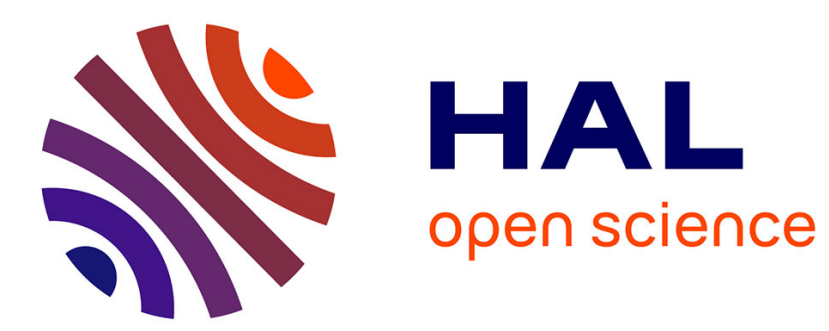

\title{
Exosomes: A Novel Therapeutic Paradigm for Treatment of Depression
}

\author{
Amanda K. A. Silva
}

\section{To cite this version:}

Amanda K. A. Silva. Exosomes: A Novel Therapeutic Paradigm for Treatment of Depression. Current Drug Targets, 2020, 21, 10.2174/1389450121999201006193005 . hal-03097048

\section{HAL Id: hal-03097048 \\ https://hal.science/hal-03097048}

Submitted on 5 Jan 2021

HAL is a multi-disciplinary open access archive for the deposit and dissemination of scientific research documents, whether they are published or not. The documents may come from teaching and research institutions in France or abroad, or from public or private research centers.
L'archive ouverte pluridisciplinaire HAL, est destinée au dépôt et à la diffusion de documents scientifiques de niveau recherche, publiés ou non, émanant des établissements d'enseignement et de recherche français ou étrangers, des laboratoires publics ou privés. 
2 Exosomes: A Novel Therapeutic Paradigm for Treatment of Depression

3 Shvetank Bhatt ${ }^{1^{*}}$, Jovita Kanoujia ${ }^{1}$, Arghya Kusum Dhar ${ }^{2}$, Surendar Arumugam ${ }^{3}$,

4 Amanda K. A. Silva ${ }^{3}$, Neeraj Mishra ${ }^{1}$

$5 \quad{ }^{1}$ Amity Institute of Pharmacy, Amity University Madhya Pradesh (AUMP), Gwalior-474005,

6 India

$7 \quad{ }^{2}$ Gurunanak Institute of Pharmaceutical Science and Technology, Kolkata-700110, India

$8{ }^{3}$ Laboratoire Matière et Systèmes Complexes (MSC), Université de Paris, UMR 7057 CNRS,

975205 Paris cedex 13, France

10 Principal Corresponding Author: *Dr. Shvetank Bhatt

11

12

Professor

Amity Institute of Pharmacy,

Amity University Madhya Pradesh (AUMP),

Gwalior-474005

Email: shvetankbhatt@gmail.com

Contact No: +919414822323

\section{Abstract}

Extracellular vesicles (EVs) of endocytic origin are known as exosomes. These vesicles are released by cells and are accessible in biofluids, such as saliva, urine, and plasma. These vesicles are made up of small RNA, DNA, proteins and play a vital role in many physiological processes. In central nervous system (CNS), they participate in various physiological processes such as stress of nerve cells, communication between the cells, synaptic plasticity and neurogenesis. The role of exosomes in depression needs to be explored further. It is known that exosomes can cross blood brain barrier (BBB), which is made up of glial cells astrocytes. One of the advantages of these vescicles is that they are able to transfer macromolecules like DNA, protein, mRNAs and miRNAs to recipient cells. This review focuses on the potential role of exosomes in depression and their utilization as atreatmentoption or diagnostic tool of depression.

Key words: Depression, Exosomes, BBB, Serotonin, Biomarker, Oxidative Stress 


\section{Introduction}

\section{Major Depressive Disorder (MDD)}

Depression or MDD is the most common mental disorder that affects more than 264 million people worldwide [1]. It is a leading cause of disability globally. Moreover, depression also increases the risk of suicide attempts [2]. Women are more susceptible to get depression as compared to men [3]. There are many psychological and pharmacological treatments available for depression and most of the treatment approaches work through modulation of the levels of bionergic amines in the CNS [4]. However, the available therapeutic approaches are limited in their role to control resistant cases of depression as well as therapeutic efficacy of medications take more time to subside the symptoms of disease [5]

There are various psychological, environmental and biological factors involved in the pathophysiology of depression [6]. Now genetic link is also found and validated. The genes related to brain derived neurotrophic factor (BDNF), 5-hydroxy tryptamine transporter (5HTT), and Norepinephrine transporter (NET) have been identified [7]. Multiple theories and hypotheses are coined to explain the pathogenesis of depression in that monoamine theory is widely acceptable. The monoamine hypothesis relies on the abnormally excessive biotransformation of major neurotransmitters (5-HT, norepinephrine and dopamine) [8]. The illustration of MDD pathogenesis involves genetic predisposition, deranged monoamine synthesis/function, and altered structure/function of brain. In addition to above mentioned factors, depression is also associated with increased oxidative stress due to the formation of reactive oxygen species (ROS) and imbalance in oxidant and antioxidant signaling [9]. Brain is more susceptible to oxidative stress due to more consumption of oxygen, higher lipid contents and weaker antioxidative defence. Dysregulation of hypothalamic pituitary adrenal (HPA) axis also associated with pathogenic progression of depression [10]. Moreover, in depression, the levels of various inflammatory markers are increased in blood and CNS area. Elevated levels of C-reactive protein and cytokines such as interleukin 6 and tumour necrosis factor-alpha were observed [11]. Treatment with anti-depressants suppresses the inflammatory response, whereas electroconvulsive therapy acutely increases the levels of various proinflammatory cytokines [12].

MDD also has strong relationship with cardiovascular disorders and is linked with high death rates [13]. Some previous reports have demonstrated that depression increases the risk of developing cardiac disease, specifically coronary artery disease, and exacerbates the 
prognosis after myocardial infarction $[13,14,15]$. Anxiety and panic disorders are another disorders found in comorbidity in approximately $80 \%$ of MDD cases. MDD also increases the risk of drug abuse and alcohol consumption [16,17].

\section{Extracellular Vesicles (EVs)}

Since there is no effective therapy available for the treatment of depression and associated comorbid disorder, innovative approaches should be investigated. In this review we will discuss the role of extracellular vesicles, including exosomes as therapeutic target and biomarker for the treatment and early detection of symptoms of depression. EVs are shed by most of the cells that release them into the extracellular space. These vesicles have very specific and important role in signaling and communication between cells. The vesicles mainly categorized in three sub types 1] apoptotic bodies (500-2000 nm); 2] microvesicles (50-1000 nm); and 3] exosomes (40-200 nm) [18]. They have different biological properties. Apoptotic bodies are engulfed by macrophages while microvesicles contain a range of cargo that is delivered to neighbouring cells. Exosomes are developed from intraluminal vesicles (ILV) via inward budding process of multivesicular body (MVB). They fuse with plasma membrane that release its ILV contents as EVs called exosomes into the extracellular space $[19,20]$. Exosomes contains proteins, lipids, and nucleic acids. They are highly enriched with microRNA (miRNA). Disease states leads to altered expression of miRNA in exosomes making the miRNA cargo intriguing candidates for investigation [21]. EVs are lipophilic in nature and easily able to cross $\mathrm{BBB}$. This specific property of exosomes makes them a target of choice for the treatment or early diagnosis of CNS disorders like depression [22,23,24].

EVs can be isolated from serum, saliva, cerebrospinal fluid, urine, breast milk, synovial fluid, effusions, semen and cell cultures [25]. The isolation of EVs is done via centrifugation at 100,000 g and/or mechanical filters, which are specifically designed according to diameter preferred EV subtype. Limited number of research studies is conducted to evaluate the role of exosomes in mental disorders.

\subsection{Blood Brain Barrier (BBB) Penetration and Extracellular Vesicles (EVs)}

Effective Delivery of drugs to the brain for the treatment of central nervous system (CNS) disorders for examples neurodegenerative diseases, tumors, trauma, stroke, autoimmune diseases is a major challenge $[27,28,29,30]$. BBB is a border that limits and regulates the passage of substances between the peripheral vascular circulation and the CNS, thereby 
1 providing protection to the CNS from toxic substances or overactive immune responses 2 [28,31]. However the BBB permits transmembrane diffusion of lipid soluble molecules of 3 size less than $400 \mathrm{Da}$ and selective transport of some compounds into and out of the brain $4 \quad[32]$

5 Two plausible transport mechanisms across the BBB were proposed namely transcellular 6 through BMECs, and paracellular through junctions between BMECs. The main components 7 of BBB are microvascular endothelial cells (BMECs), astrocytes, pericytes, the endothelial 8 basement membrane, and neighbouring neurons. A complex arrangement of tight junctions 9 (TJs) and adherens junctions (AJs) in the brain endothelial cells regulates paracellular permeability [33].

- Around $98 \%$ of small molecule drugs and almost all of large molecule biologic drugs, including recombinant proteins, monoclonal antibodies, or gene-based medicines are unable to cross the BBB $[34,35]$. Exosomes with its contents are capable of crossing the BBB. This finding has promoted biomarker research with exosomes and their use as a drug delivery system. Numerous studies have demonstrated the successful brain delivery of exosomes. Effective delivery of GAPDH siRNA to the brain via systemic injection of exosomes in mice [36] was reported. Other experiments have been proved to be successful in delivering exosomes to the mice brain via intranasal injection [37]. In another experiment using rats, a fluorescently tagged protein selectively expressed in rat brain tissue was recovered in small EVs having same characteristics as exosomes in their blood [38]. These studies support the fact that exosomes are able to communicate from the brain to the rest of the body and they can cross the $\mathrm{BBB}$ in a bi-directional manner. However, their exact mechanism of BBB penetration remains unclear.

In an experiment using in vitro $\mathrm{BBB}$ model the transfer of EVs derived from human erythrocytes was found to be dependent on the adsorptive-mediated transcytosis method of transport [39]. Though the EVs crossed under healthy and inflammatory conditions, the peripheral administration of lipopolysaccharides was found to considerably increase the EVs movement across the BBB [39]. Another study revealed that in healthy and stroke-like condition exosomes crossed a BBB model using transcellular BMEC endocytosis, suggesting that exosomes maintain their ability to cross during stressful conditions [40]. This same study also indicated that exosomes were internalized through endocytosis, and accumulate in endosomes. There was a decrease in 
exosome transcellular migration using chlorpromazine (CPZ) an inhibitor for clathrindependent endocytosis, which transfers clathrin from the surface of cells to intracellular endosomes $[40,41]$. This indicates that clathrin-dependent endocytosis may be involved in transportation of exosomes across the BBB [40].

Studies have indicated that methyl- $\beta$-cyclodextrin $(\mathrm{M} \beta \mathrm{CD})$, which removes cholesterol from the plasma membrane [41], and filipin III, which binds to cholesterol caused a significant reduction in BBB penetration of exosomes [40]. This result revealed caveolae-dependent endocytosis as alternative route of exosome migration. Most likely the uptake of exosomes in BMEC depends on specific ligand receptors or lipid rafts, and mechanisms of exosome uptake may also depend on the cell for which they originate. It has been postulated that exosomes from different cell origins have different content including protein and lipids, possibly modifying their mechanism of BBB penetration [42]. Moreover, disease condition may influence the mechanism of BBB crossing, as the contents of exosomes change upon disease state $[26,42]$.

Studies have also proposed that exosomes play a role in increased permeability of vascular barriers of the BBB. Breast cancer cells secreted exosomes uniquely expressed miR-105, which directly targeted the tight junction protein ZO-1 [43]. This exosome transfer of miR105 destroyed tight junctions and the integrity of the BBB [43]. Moreover, claudin-5 (Cldn5) a tight junction protein of the BBB was found to be encapsulated in exosomes [44]. A loosened BBB was found in the Cldn5 knockout mice [45], suggested that exosomes carrying Cldn5 might play a role in BBB integrity. It was also found that a decline in Cldn5 induced depressive-like behaviors in mice, and antidepressant treatment increased Cldn5 levels and promoted recovery [46]. A leaky BBB has been linked to neuroinflammation $[47,48]$. Thus, the possible influence of exosomes on the BBB integrity also advocate a role for exosomes in neuroinflammation and the pathogenesis of mental disorders [47,48] Hence, in mental disorders a leaky BBB state may be caused by exosomes released from cells being influenced by this disease state.

\subsection{Signalling mechanisms involving exosomes in the brain}

An important role is played by exosomes in cell to cell communication in the CNS..They are involved in signalling through distal as well as neighbouring cells [49]. The exosomes work as an important link for communication with similar kind of cells or also with different types 
1 of cells. The release of exosomes in the CNS is controlled by glutaminergic neurons and influx of Calcium ions [50,51].

3 N-methyl-D-aspartate (NMDA) is a glutaminergic receptor. The entry of calcium ion through 4 gates of this receptor triggers the neuronal release of exosomes. Other important 5 glutaminergic receptor which is involved in physiological role of exosomes is and $\alpha$-amino-36 hydroxy-5-methyl-4-isoxazolepropionic acid (AMPA) receptors [50]. After releasing, 7 exosomes can fuse with cell membrane of the recipient cell and release its constituents inside 8 the cytoplasm of the cell, transfer lipid and protein content on the cell surface. Internalisation of exosomes can also take place by macropinocytosis or endocytosis process. The fusion of exosomes is regulated by receptor mediated mechanisms. These diverse mechanisms for transfer of constituents can initiate different signaling mechanisms in recipient cells [52].

Serotonin or 5-hydroxy tryptamine $(5-\mathrm{HT})$ has also substantial role on the release of exosomes from the non-neuronal brain cells such as microglial cells. 5-HT is a major neurotransmitter which has important role in the pathophysiology of depression. According to monoamine theory level of 5-HT is decreased in depression and bipolar disorders [53]. Serotonin can increase cytosolic levels of calcium ions, which leads to stimulation of release of exosomes from primary microglia [51]. Since 5-HT is involved in release of exosomes, their release is modified predominantly in case of depression. Both the factors discussed above i.e. cell to cell communication and neurotransmitter mediated release of exosomes are important aspects of psychopharmacology and involved in various CNS disorders including depression [54]. So exosomes can be considered to play a major role in etiopathogenesis of mental disorders given their prominence in the regulation of cell communication, and their regulation via neurotransmitters. In addition, signaling through one nerve cell to other nerve cell is important in synaptic plasticity [55].

\section{Role of Exosomes in Depression}

Depression is an important psychiatric disorder which is associated with disability and affects person daily life. Diagnostic and statistical manual of mental disorders, 4th edition (DSM-IV) has given 9 symptoms of depression out of which if 5 symptoms are present for more than two weeks then patient can be considered as depressed. However, these have certain limitations and more evaluation is required to confirm the depression cases. Multiple evidence gathered from previous research suggested that estimation of various biomarkers could be a useful approach for early detection of depression. Limited number of research 
1 studies is conducted to evaluate the role of exosomes in mental disorders $[56,57,58)$

2 Particulary, Banigan et al. investigated exosomes from frozen postmortem prefrontal cortex

3 to study alterations in miRNA in patients of psychoses and bipolar disorder. They found that

$4 \quad \mathrm{miR}-497$ in psychoses patients and miR-29c in bipolar patients to be upregulated compared to

5 control subjects [26]. This early piece work opens up interesting opportunities to study

6 exosomes in mental disorders, demonstrating that miRNA cargo may be interesting to be

7 evaluated in these phenotypes. The intercellular signalling of exosomes has important role in

8 normal physiological and pathological conditions in the CNS. For example glial cell

9 oligodendrocyte-derived exosomes are thought to be involved in neuroprotection against cellular stress. The reason of this protective effect of exosomes is due to involvement of proteins released from exosomes inside the recipient cell $[56,59,60]$.

Recent data on signaling of exosomes in the CNS highlighted their role in transcriptional regulation, neurogenesis, plasticity, and neuroinflammation [60]. Exosomes delivered intravenously can be demonstrated to cross the BBB naturally. Exosomes from mesenchymal stem cells (MSC's) demonstrate anti-inflammatory and pro-growth effects in preclinical models and clinical cases reports and have been used intravenously and with intracerebral and intrathecal injection..

Among various biomarkers microRNAs (miRNAs) emerged as an important diagnostic marker that could be beneficial for patients to detect depression. These biomarkers have predominant role in pathophysiology of depression [61]. These molecules affect various molecular levels signaling pathways involved in progression of depression. miRNAs could be used as diagnostic and therapeutic biomarkers in MDD patients. Besides miRNAs, exosomes as nano- carriers could have been emerged as diagnostic biomarkers in various diseases such as MDD and other CNS disorders. Intravenous delivery of exosomes can able to cross the BBB [62]. Exosomes and MSC's demonstrate anti-inflammatory and pro-growth effects in preclinical models and clinical cases.

The exosomes released from mast cells have protective effect against increased oxidative stress. They have capacity to communicate a protective signal to other cells which are exposed to oxidative stress and this leads to reduction in cell death [63]. Exosomes that are produced by one cell under stress condition is able to induce capacity to tolerate increased oxidative stress in another cell that is known as recipient cell. This effect is linked with changed exosomal mRNA content that can be attenuated by reduced RNA activity through 
exposure if UV light $[64,65]$. EVs and ROS are closely linked with oxidative stress. The prooxidant conditions are involved in release of EVs in fact, NADPH oxidase and nitric oxide synthase-2 (NOS-2) inhibitors inhibit the production of EVs in neutrophils [66,67]. According to a study conducted by Wei ZX 2020 Exosomes obtained from patients with MDD caused depressive-like behaviors in mice with involvement of micro RNA regulated neurogenesis.

Intravenous injection of Exosomes derived from blood of MDD patients into normal mice exhibited the depressive phenotype behaviors as evaluated by the battery of behavioral tests such as forced swim test, tail suspension test, and novelty suppressed feeding tests, and these behavious reversed by administration of exosomes derived from healthy subjects into chronic unpredictable mild stress (CUMS)-treated mice. [68]. In general, secretion of exosomes is increased in response to stress or pathological conditions [69].

.Various recent studies have observed changes in miRNAs in depressed patients, which target not only important pathways associated with memory function, synaptic plasticity but also formation of neurotrophic factors and modulation of immune cells[70,71]. Transfer of EVs or exosomes to nerve cells occurs through supporting cells of nervous system such as oligodendrocytes, microglia and astrocytes. Besides their potential as biomarkers, these exososmes have a predominant role in pathophysiology and dissemination of inflammatory pathways [72]. Reactive microglia were shown to release exosomes and microvesicles (MVs)

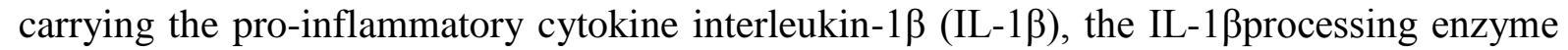
caspase-1, and the $\mathrm{P} 2 \mathrm{X} 7$ receptor that may induce and propagate inflammatory reactions throughout the brain [73]. Additionally, monocytes that are activated by interferon alpha and/or lipopolysaccharides (LPS release exosomes that carry altered miRNA profiles as shown in Fig. 1.

A study data published in the Journal of Affective Disorders indicates a link between higher levels of certain neuron-derived exosomes (NDE) and MDD [74]. In addition, a clinical study has been started to evaluate the safety and efficacy of exosomes against refractory depression [75]. Impaired adult hippocampal neurogenesis (AHN) is associated with depression and other neurodegenerative disorders like schizophrenia [76]. Protein analysis study reveals that exosomes has important role in modulation of adult neurogenesis [76]. In addition, injection of known pathogen containing exosomes into the dentate gyrus is sufficient to impair AHN in mice [77]. Cytokines and corticosteroids stimulate the release of glial cell astrocyte related 
exosomes which contains several miRNAa that are critical for neurogenesis, stress response and survival of neurons [76]. So the exosomes may have dual role as it may be involved in maintenance and inhibition of neurogenesis in adult. Nerve cell to supporting glial cell signalling is one of the important mechanisms for exosomes [78]. Injection of exosomes in the tail vein of immune-challenged mice leads to increase in CNS expression of proinflammatory cytokine mRNA and associated miRNA in mice. Protein analysis of exosomes in the CNS reveals cargo involved in modulating adult neurogenesis [79].

\section{Exosomes as Biomarker in CNS diseases and depression}

Exosomes have immense capability as diagnostic tool or biomarker for early diagnosis of CNS disorders such as Depression and Schizophrenia. Pathophysiological changes in contents of exosomes have been observed in CNS disorders. The exosomes can be isolated without much difficulty from biological fluids including urine, saliva and blood [80]. Moreover exosomes contents are not easily degradable by biological fluids or enzyme in our body as they are protected inside the membrane [54]. In addition, ex vivo, these exosomes are stable for longer duration can be stored long time before analysis. It is possible to trace the surface marker indicating the origin for exosomes. The exosomes have great potential to cross BBB and this property makes them a good biomarker to identify a CNS disorder [81]. Of particular interest is the ability to characterize exosomes based on their cell of origin, potentially providing an extra layer of insight into the disease of interest [82]. The significance of exosomes as diagnostic tool in disease like cancer is already established. However their role in CNS disorders like depression is yet to be established. Many of the circulating proteins and nucleic acids are diluted in the blood stream and the majority of them originate from other sources besides neurons due to the tight regulation of BBB in molecule transport.

Different types of exosomes (from multiple cells) can be segregated from biofluids using different segregation techniques like ultracentrifugation, immunomagnetic beads, and chromatography $[83,84]$. In addition, treatment with RNase, western blot analysis or mass spectrometry is also used for identification/segregation of exosomes contents. Various types of exosomes can be isolated from biofluids using multiple methods. Additionally, exosomes have phospholipid bilayer; therefore treatment with RNase prior to use will ensure that cargo used downstream was encapsulated within the vesicle [85]. Various types of exosomes may be identified using western blots or mass spectrometry of proteins which are involved in 
biogenesis of ILVs, including tetraspanins and proteins involved in the ESCRT machinery needed for biogenesis [86]. It is essential to remember that these markers are not exclusively associated with exosomes and further characterizations of these markers are required..

Exosomes derived from developing and mature neurons of hippocampus, contain L1 cell adhesion molecule, and the GluR2/3 subunits of glutamate receptors, both of which are well established markers of nerve cells [87,88]. Protein markers, such as glial fibrillary acidic protein), glutamine aspartate transporter, and glutamine synthetase, can be used to enrich for astrocytic-derived exosomes. Additionally, myelin proteo-lipid protein and 2', 3'-cyclic nucleotide 3'-phosphodiesterase have been identified on oligodendrocytes derived from exosomes [89] . Neural-derived exosomes from plasma have also been used in a small study to evaluate protein biomarkers for patients with MDD [71]. The expression levels of exosomal miRNAs vary among different biological fluids in vivo and culture medium in vitro. So these changes in expression of exosomes in MDD are used to identify the early stage of disorder.

\section{Authors insight on the topic}

Utilization of EVs as drug carrier has been well explored in chronic disorders such as cancer and autoimmune disorders. In the future, drug loaded EVs could be explored to achieve maximum therapeutic benefit sparing normal healthy cells, with less off target mediated adverse effects of antidepressants with aim to target the selective affected region owing to MDD induced alteration in the CNS. Disorders like depression have lack of suitable diagnostic technique and treatment resistance is also more common. So, EVs maybe serve as as a potential therapeutic candidate as well as biomarker, which may be effective in the field of depression. For that, further studies have to be performed in order to understand the homing of EVs from different cell types in MDD. In MDD, it has been documented that in addition to abnormal neurotransmitter biogenesis and neurotransmitter shuttling, involvement of neuronal damage is highly prevalent condition during later stage of pathophysiological mechanism to disease progression. Neurogenesis capability of currently available antidepressants alone or in combination are being explored and evaluated in preclinical and clinical experiments. However, regenerative potential of these agents is not substantial to protect the vigorous constant neurodegenerative process during later stage of MDD and associated conditions. As it has been witnessed that EVs have regenerative ability and this exclusive property of EVs can be exploited to prevent the ongoing neurodegenerative process 
1 of MDD. Cell therapy-based treatment especially induced pluripotent stem cells exhibit 2 therapeutic activity in MDD and associated pathological conditions, however its usage for 3 these conditions have been limited by its adverse effects such as graft rejection by recipient, 4 immunogenicity reactions and low accessible and penetration to $\mathrm{BBB}$. There are various 5 approaches being explored to mitigate these adverse effects caused by cell therapy. In this 6 context, there are advantages of EVs compared to cells such as reduced size, less 7 immunogenicity, easier accessibility to BBB along with carrying some essential components 8 of cell while mimicking benefits elicited by cell therapy of MDD. This lays foundation for 9 cell-free therapy for MDD associated pathological condition. Despite the expected EV benefits for treating MDD, there are still unexplored uncertainties in terms of precise pharmacological mechanism of action, route of administration.

Selectivity and accumulation of EV to intended target site of action will be hampering the effort of EV as therapeutic potential in treating MDD. Though selective binding to its target can be achieved by producing EV which has selective binding surface expression molecules on its surface from genetically modified parental cells expressing the target selection marker. There are plenty of invitro cell-based assays need to be performed to achieve the tissue and target selective EV to maximize its therapeutic benefit sans adverse effect on healthy brain tissues. Another obstacle to developing EV for MDD would be owing to plethora of mechanism of action elicited by EV, narrow downing a precise pharmacological molecular mechanism action can be difficult and challenging task. Nevertheless, the MDD disease pathway related molecular markers could be selected to elucidate the prime mode of mechanism action of EVs are warranted using various molecule biology tools (reporter, knock out or knock in based approaches. To address these issues, initiation of well-planned preclinical studies including high translational value based in vitro (Target binding, specificity, selectivity and internalization assays), pharmacokinetic assays (Tissue distribution studies with special emphasis on central nervous system using appropriate labelling agents) in vivo models of MDD, toxicology and safety pharmcology studies on vital systems should be warranted. In conclusion, owing to better advantage of EV compared to conventional treatment, future research EVs based therapy is expected to provide evidence on their potential as standalone therapy or in combination with standard antidepressants in the management of this devastating disease. 
2 In the future, EVs may be useful as effective therapeutic carriers as well as biomarkers for the

\section{Conclusion} diagnosis of depression in early stages. However, further studies need to be done to prove the efficacy and pharmacokinetic profile of these exosomes. The exosomes have an advantage over conventional treatment to able to cross the BBB. In depression modulation in levels of exosomes is taking place and this can be used as marker for detection of disorder in early stages. Chronic stress and inflammation also lead to modify the expression of exosomes. Exosomes are important in communication and signalling between two cells. These EVs also have role in synaptic plasticity and neurogenesis. However, some more detailed studies are required to validate the role of exosomes in MDD. In the future, exosomes may be used as effective treatment and biomarker for identification of depression and other CNS disorders.

Conflict of Interest: There is no conflict of interest among the authors.

\section{Acknowledgement: None}

Financial Assistance: Nil

\section{References}

1. https://www.who.int/news-room/fact-sheets/detail/depression

2. Brådvik, L. Suicide Risk and Mental Disorders. International journal of environmental research and public health, 2018, 15(9), 2028.

3. Albert, P. R. Why is depression more prevalent in women? Journal of psychiatry \& neuroscience, 2015, 40(4), 219-221.

4. Heninger ,G.R.; Delgado, P.L.; Charney, D.S. The revised monoamine theory of depression: a modulatory role for monoamines, based on new findings from monoamine depletion experiments in humans. Pharmacopsychiatry, 1996, 29(1), 211.

5. Al-Harbi, K. S. Treatment-resistant depression: therapeutic trends, challenges, and future directions. Patient preference and adherence, 2012, 6, 369-388.

6. Hasler, G. Pathophysiology of depression: do we have any solid evidence of interest to clinicians? World psychiatry, 2010, 9(3), 155-161.

7. Tamatam, A.; Khanum, F.; Bawa, A. S. Genetic biomarkers of depression. Indian journal of human genetics, 2012, 18(1), 20-33. 
8. Liu, B.; Liu, J.; Wang, M.; Zhang, Y.; Li, L. From Serotonin to Neuroplasticity: Evolvement of Theories for Major Depressive Disorder. Frontiers in cellular neuroscience, 2017, 11, 305.

9. Bhatt, S.; Radhakrishnan, M.; Jindal, A.; Devadoss, T.; Dhar, A. K. Neuropharmacological evaluation of a novel 5-HT3 receptor antagonist (6g) on chronic unpredictable mild stress-induced changes in behavioural and brain oxidative stress parameters in mice. Indian journal of pharmacology, 2014, 46(2), 191-196.

10. Keller, J.; Gomez, R.; Williams, G.; Lembke, A.; Lazzeroni, L.; Murphy, G. M. Jr.; Schatzberg, A. F. HPA axis in major depression: cortisol, clinical symptomatology and genetic variation predict cognition. Molecular psychiatry, 2017, 22(4), 527-536.

11. Lee, C. H.; Giuliani, F. The Role of Inflammation in Depression and Fatigue. Frontiers in immunology, 2019, 10, 1696.

12. Dinan,T.G. Inflammatory markers in depression. Curr Opin Psychiatry, 2009, 22(1), 32-6.

13. Dhar, A. K.; Barton, D. A. Depression and the link with cardiovascular disease. Frontiers in psychiatry, 2016, 7, 33.

14. Mastrogiannis, D.; Giamouzis, G.; Dardiotis, E.; Karayannis, G.; Chroub-Papavaiou, A.; Kremeti, D.; Spiliopoulos, K.; Georgoulias, P.; Koutsias, S.; Bonotis, K.; Mantzorou, M.; Skoularigis, J.; Hadjigeorgiou, G. M.; Butler, J.; \& Triposkiadis, F. (2012). Depression in patients with cardiovascular disease. Cardiology research and practice, 2012, 794762.

15. Strik, J.J.; Honig, A.; Maes M. Depression and myocardial infarction: relationship between heart and mind. Prog Neuropsychopharmacol Biol Psychiatry, 2001, 25(4), 879-92.

16. Burns, L.; Teesson, M.; O'Neill, K. The impact of comorbid anxiety and depression on alcohol treatment outcomes. Addiction. 2005, 100(6), 787-96.

17. Hirschfeld, R. M. The Comorbidity of Major Depression and Anxiety Disorders: Recognition and Management in Primary Care. Primary care companion to the Journal of clinical psychiatry, 2001, 3(6), 244-254.

18. Gheinani, A.H.; Vögeli, M.; Baumgartner, U.; Vassella, E.; Draeger, A.; Burkhard, F.C.; Monastyrskaya, K. Improved isolation strategies to increase the yield and purity of human urinary exosomes for biomarker discovery. Scientific Reports, 2018, $8(1), 3945$.

19. Lee, Y.; El Andaloussi, S.; Wood, M. J. A. Exosomes and microvesicles: extracellular vesicles for genetic information transfer and gene therapy. Human Molecular Genetics 2012 21(R1), R125-R134.

20. Johnstone, R. M.; Adam, M.; Hammond, J. R.; Orr, L.; Turbide, C. Vesicle formation during reticulocyte maturation. Association of plasma membrane activities with released vesicles (exosomes). Journal of Biological Chemistry, 1987, 262, 9412 9420.

21. Gallo, A.; Tandon, M.; Alevizos, I.; \& Illei, G. G. The majority of microRNAs detectable in serum and saliva is concentrated in exosomes. PLOS ONE, 2012, 7 , e30679. 
22. Chen, C.C.; Liu, L.; Ma, F.; Wong, C.W.; Guo, X.E.; Chacko, J.V.; Farhoodi, H.P.; Zhang, S.X.; Zimak, J.; Ségaliny, A.; Riazifar, M.;Pham, V.; Digman, M.A.; Pone, E.J.; Zhao, W. Elucidation of Exosome Migration across the Blood-Brain Barrier Model In Vitro. Cell Mol Bioeng, 2016, 9(4), 509-529.

23. Cufaro, M. C.; Pieragostino, D.; Lanuti, P.; Rossi, C.; Cicalini, I.; Federici, L.; De Laurenzi, V.; Del Boccio, P. Extracellular Vesicles and Their Potential Use in Monitoring Cancer Progression and Therapy: The Contribution of Proteomics. Journal of oncology, 2019, 2019, 1639854.

24. Yao, Z. Y., Chen, W. B., Shao, S. S., Ma, S. Z., Yang, C. B., Li, M. Z., Zhao, J. J., Gao, L.. Role of exosome-associated microRNA in diagnostic and therapeutic applications to metabolic disorders. Journal of Zhejiang University. Science. 2018, 19(3), 183-198.

25. Zebrowska, A.; Skowronek, A.; Wojakowska, A.; Widlak, P.; Pietrowska, M. (2019). Metabolome of Exosomes: Focus on Vesicles Released by Cancer Cells and Present in Human Body Fluids. International journal of molecular sciences, 2019, 20(14), 3461.

26. Banigan, M. G.; Kao, P. F.; Kozubek, J. A.; Winslow, A. R.; Medina, J.; Costa, J.; Schmitt, A.; Schneider, A.; Cabral, H.; Cagsal-Getkin, O.; Vanderburg, C. R.; Delalle, I. Differential expression of exosomal microRNAs in prefrontal cortices of schizophrenia and bipolar disorder patients. PloS one, 2013, 8(1), e48814.

27. Abbott, N.J.; Rönnbäck, L.; Hansson, E. Astrocyte-endothelial interactions at the blood-brain barrier. Nature Reviews Neuroscience, 2006, 7(1), 41-53.

28. Obermeier, B.; Daneman, R.; Ransohoff, R.M. Development, maintenance and disruption of the blood-brain barrier. Nature Medicine, 2013, 19(12), 1584-96.

29. Rubin, L.L.; Staddon, J.M. The cell biology of the blood-brain barrier. Annual Review of Neuroscience, 1999, 22, 11-28.

30. Upadhyay, R.K. Drug delivery systems, CNS protection, and the blood brain barrier. BioMed Research International, 2014, 2014, 869269.

31. Andreone, B.J.; Lacoste, B.; Gu, C. Neuronal and vascular interactions. Annual Review of Neuroscience. 2015, 38, 25-46.

32. Sanchez-Covarrubias, L.; Slosky, L. M.; Thompson, B. J.; Davis, T. P.; Ronaldson, P. T. Transporters at CNS barrier sites: obstacles or opportunities for drug delivery? Current Pharmaceutical Design. 2014, 20(10), 1422-49.

33. Wolburg, H.; Lippoldt, A. Tight junctions of the blood-brain barrier: development, composition and regulation. Vascular Pharmacology. 2002, 38(6), 323-37.

34. Pardridge, W.M. Drug transport across the blood-brain barrier. Journal of Cerebral Blood Flow \& Metabolism. 2012, 32(11), 1959-72.

35. Pardridge, W.M. The blood-brain barrier: bottleneck in brain drug development. NeuroRx. 2005, 2(1), 3-14.

36. Alvarez-Erviti, L.; Seow, Y.; Yin, H.; Betts, C.; Lakhal, S.; Wood, M.J. Delivery of siRNA to the mouse brain by systemic injection of targeted exosomes. Nature Biotechnology. 2011, 29(4), 341-5.

37. Zhuang, X.; Xiang, X.; Grizzle, W.; Sun, D.; Zhang, S.; Axtell, R.C.; Ju, S.; Mu, J.; Zhang, L.; Steinman, L.; Miller, D.; Zhang, H.G. Treatment of brain inflammatory 
diseases by delivering exosome encapsulated anti-inflammatory drugs from the nasal region to the brain. Molecular Therapy. 2011, 19(10), 1769-79.

38. Gómez-Molina, C.; Sandoval, M.; Henzi, R.; Ramírez, J.P.; Varas-Godoy, M.; Luarte, A.; Lafourcade, C.A.; Lopez-Verrilli, A.; Smalla, K.H.; Kaehne, T.; Wyneken, U. Small Extracellular Vesicles in Rat Serum Contain Astrocyte-Derived Protein Biomarkers of Repetitive Stress. International Journal of Neuropsychopharmacology. 2019, 22(3), 232-246.

39. Matsumoto, J.; Stewart, T.; Sheng, L.; Li, N.; Bullock, K.; Song, N.; Shi, M.; Banks, W.A.; Zhang, J. Transmission of $\alpha$-synuclein-containing erythrocyte-derived extracellular vesicles across the blood-brain barrier via adsorptive mediated transcytosis: another mechanism for initiation and progression of Parkinson's disease? Acta Neuropathologica Communications. 2017, 5(1), 71.

40. Chen, C.C.; Liu, L.; Ma. F.; Wong, C.W.; Guo, X.E.; Chacko, J.V.; Farhoodi, H.P.; Zhang, S.X.; Zimak, J.; Ségaliny, A.; Riazifar, M.; Pham, V.; Digman, M.A.; Pone, E.J.; Zhao, W. Elucidation of Exosome Migration across the Blood-Brain Barrier Model In Vitro. Cellular and Molecular Bioengineering. 2016, 9(4), 509-529.

41. Dutta, D.; Donaldson, J.G. Search for inhibitors of endocytosis: Intended specificity and unintended consequences. Cellular Logistics. 2012, 2(4), 203-208.

42. Haraszti, R.A.; Didiot MC, Sapp E, Leszyk J, Shaffer SA, Rockwell HE, Gao F, Narain NR, DiFiglia M, Kiebish MA, Aronin N, Khvorova A. High-resolution proteomic and lipidomic analysis of exosomes and microvesicles from different cell sources. Journal Extracellular Vesicles. 2016, 17(5), 32570.

43. Zhou, W.; Fong, MY.; Min, Y.; Somlo, G.; Liu, L.; Palomares, M.R.; Yu, Y.; Chow, A.; O'Connor, S.T.; Chin, A.R.; Yen, Y.; Wang, Y.; Marcusson, E.G.; Chu, P.; Wu, J.; Wu, X.; Li, A.X.; Li, Z.; Gao, H.; Ren, X.; Boldin, M.P.; Lin, P.C.; Wang, S.E. Cancer-secreted miR-105 destroys vascular endothelial barriers to promote metastasis. Cancer Cell. 2014, 25(4), 501-15.

44. Paul, D.; Baena, V.; Ge, S.; Jiang, X.; Jellison, E.R.; Kiprono, T.; Agalliu, D.; Pachter, J.S. Appearance of claudin-5(+) leukocytes in the central nervous system during neuroinflammation: a novel role for endothelial-derived extracellular vesicles. Journal of Neuroinflammation. 2016, 13(1), 292.

45. Nitta, T.; Hata, M.; Gotoh, S.; Seo, Y.; Sasaki, H.; Hashimoto, N.; Furuse, M.; Tsukita, S. Size-selective loosening of the blood-brain barrier in claudin-5-deficient mice. Journal of Cell Biology. 2003,161(3), 653-60.

46. Menard, C.; Pfau, M.L.; Hodes, G.E.; Kana, V.; Wang, V.X.; Bouchard, S.; Takahashi, A.; Flanigan, M.E.; Aleyasin, H.; LeClair, K.B.; Janssen, W.G.; Labonté, B.; Parise, E.M.; Lorsch, Z.S.; Golden, S.A.; Heshmati, M.; Tamminga, C.; Turecki, G.; Campbell, M.; Fayad, Z.A.; Tang, C.Y.; Merad, M.; Russo, S.J. Social stress induces neurovascular pathology promoting depression. Nature Neuroscience. 2017, 20(12), 1752-1760.

47. Maes, M. The cytokine hypothesis of depression: inflammation, oxidative \& nitrosative stress (IO\&NS) and leaky gut as new targets for adjunctive treatments in depression. Neuro Enocrinology Letters. 2008, 29(3), 287-91. 
48. Najjar, S.; Pearlman, D.M.; Alper, K.; Najjar, A.; Devinsky, O. Neuroinflammation and psychiatric illness. Journal of Neuroinflammation. 2013, 10, 43

49. Zhang, G.; Yang, P. A. novel cell- cell communication mechanism in the nervous system: exosomes. Journal of Neuroscience Research 2018, 96, 45-52.

50. Lachenal, G.; Pernet-Gallay, K.; Chivet, M.; Hemming, F.J.; Belly, A.; Bodon, G.; Blot, B.; Haase, G.; Goldberg, Y.; Sadoul, R. Release of exosomes from differentiated neurons and its regulation by synaptic glutamatergic activity. Molecular and Cellular Neurosciences, 2011, 46(2), 409-18.

51. Glebov, K.; Löchner, M.; Jabs, R.; Lau, T.; Merkel, O.; Schloss, P.; Steinhäuser, C.; Walter, J. Serotonin stimulates secretion of exosomes from microglia cells. Glia, 2015; 63(4):626-34.

52. Sharma, P.; Schiapparelli, L.; Cline, H.T.; Exosomes function in cell-cell communication during brain circuit development. Curr Opin Neurobiol, 2013,23(6),997-1004.

53. Cowen, P. J.; Browning, M. What has serotonin to do with depression?. World psychiatry: official journal of the World Psychiatric Association (WPA), 2015, 14(2), 158-160.

54. Kanninen, K.M.; Bister, N.; Koistinaho, J.; Malm, T. Exosomes as new diagnostic tools in CNS diseases. Biochimica et Biophysica Acta, 2016, 1862(3), 403-10.

55. Chivet, M.; Hemming, F.; Fraboulet, S.; Sadoul, R. Emerging role of neuronal exosomes in the central nervous system. Frontiers in Physiology, 2012 3, 145.

56. Saeedi, S.; Israel, S.; Nagy, C.; Turecki, G. The emerging role of exosomes in mental disorders. Translational Psychiatry, 2019, 9, 122.

57. Brites, D.; Fernandes, A. Neuroinflammation and Depression: Microglia Activation, Extracellular Microvesicles and microRNA Dysregulation. Frontiers in cellular neuroscience. 2015, 9, 476.

58. Gruzdev, S.K.; Yakovlev, A.A.; Druzhkova, T.A.. The Missing Link: How Exosomes and miRNAs can Help in Bridging Psychiatry and Molecular Biology in the Context of Depression, Bipolar Disorder and Schizophrenia. Cell Mol Neurobiol, 2019, 39, $729-750$.

59. Frühbeis, C.; Fröhlich, D.; Kuo, W. P.; Amphornrat, J.; Thilemann, S.; Saab, A. S.. Neurotransmitter-triggered transfer of exosomes mediates oligodendrocyte-neuron communication. PLoS Biology, 2013, 11, e1001604.

60. Krämer-Albers, E.M.; Bretz, N.; Tenzer, S.; Winterstein, C.; Möbius, W.; Berger, H.; Nave, K.A.; Schild, H.; Trotter J. Oligodendrocytes secrete exosomes containing major myelin and stress-protective proteins: Trophic support for axons? Proteomics Clinical Applications, 2007, 1(11), 1446-61.

61. Tavakolizadeh, J.; Roshanaei, K.; Salmaninejad, A.; Yari, R.; Nahand, J.S.; Sarkarizi, H.K.; Mousavi, S.M.; Salarinia, R.; Rahmati, M.; Mousavi, S.F.; Mokhtari, R.; Mirzaei, H.; MicroRNAs and Exosomes in depression: Potential diagnostic biomarkers. J Cell Biochem, 2018, 119(5), 3783-3797.

62. Yuan, D.; Zhao, Y.; Banks, W. A.; Bullock, K. M.; Haney, M.; Batrakova, E.; Kabanov, A. V. Macrophage exosomes as natural nanocarriers for protein delivery to inflamed brain. Biomaterials, 2017, 142, 1-12. 
63. Eldh, M.; Ekström, K.; Valadi, H.; Sjöstrand, M.; Olsson, B.; Jernås, M.; Lötvall, J. Exosomes communicate protective messages during oxidative stress; possible role of exosomal shuttle RNA. PloS one, 2010 5(12), e15353.

64. Rule Wigginton, K.; Menin, L.; Montoya, J.P.; Kohn, T. Oxidation of Virus Proteins during UV254 and Singlet Oxygen Mediated Inactivation. Environmental Science \& Technology 2010,44, 5437-5443.

65. Greenberg, J.R. Ultraviolet light-induced crosslinking of mRNA to proteins. Nucleic Acids Research 1979;6:715-732.

66. Benedikter, B.J.; Weseler, A.R.; Wouters, E.F.M.; Savelkoul, P.H.M.; Rohde, G.G.U.; Stassen, F.R.M. Redox-dependent thiol modifications: implications for the release of extracellular vesicles. Cellular and Molecular Life Sciences, 2018, 75(13), 2321-2337.

67. Thom, S.R.; Bhopale, V.M.; Yang, M. Neutrophils generate microparticles during exposure to inert gases due to cytoskeletal oxidative stress. The Journal of Biological Chemistry, 2014,289(27),18831-18845.

68. Wei, Z.X.; Xie, G.J.; Mao, X.; Zou, X.P.; Liao, Y.J.; Liu, Q.S.; Wang, H, Cheng Y. Exosomes from patients with major depression cause depressive-like behaviors in mice with involvement of miR-139-5p-regulated neurogenesis. Neuropsychopharmacology. 2020, 45(6),1050-1058.

69. Ailawadi, S.; Wang, X.; Gu, H.; Fan, G.C. Pathologic function and therapeutic potential of exosomes in cardiovascular disease. Biochim Biophys Acta, 2015 , 1852(1), 1-11.

70. Donlin-Asp, PG; Polisseni, C; Klimek, R; Heckel, A; Schuman EM Differential regulation of local mRNA dynamics and translation following long-term potentiation and depression. bioRxiv. doi: https://doi.org/10.1101/2020.07.08.192369

71. Cajigas IJ, Will T, Schuman EM. Protein homeostasis and synaptic plasticity. EMBO J. 2010;29(16):2746-2752..

72. Buzas, E. I.; György, B.; Nagy, G.; Falus, A.; Gay, S. Emerging role of extracellular vesicles in inflammatory diseases. Nature Reviews Rheumatology, 2014, 10, 356-364.

73. Frühbeis, C.; Fröhlich, D.; Krämer-Albers, E. M. (2012). Emerging roles of exosomes in neuron-glia communication. Frontiers in Physiology, 2012, 3, 119.

74. Kuwano, N.; Kato, T.A.; Mitsuhashi, M.; Sato-Kasai, M.; Shimokawa, N.; Hayakawa, K.; Ohgidani, M.; Sagata, N.; Kubo, H.; Sakurai, T.; Kanba S. Neuron-related blood inflammatory markers as an objective evaluation tool for major depressive disorder: An exploratory pilot case-control study. Journal of Affective Disorders. 2018, 240, 88-98.

75. https://clinicaltrials.gov/ct2/show/NCT04202770

76. Luarte, A.; Cisternas, P.; Caviedes, A.; Batiz, L.F.; Lafourcade, C.; Wyneken, U.; Henzi, R.; Astrocytes at the Hub of the Stress Response: Potential Modulation of Neurogenesis by miRNAs in Astrocyte-Derived Exosomes. Stem Cells International, 2017, 2017, 1719050.

77. Zheng, T.; Pu, J.; Chen, Y.; Guo, Z.; Pan, H.; Zhang, L.; Zhang, H.; Sun, B.; Zhang, B. Exosomes Secreted from HEK293-APP Swe/Ind Cells Impair the Hippocampal Neurogenesis. Neurotoxicity Research, 2017, 32(1), 82-93. 
78. Bahrini, I.; Song, J-h.; Diez, D.; Hanayama, R. Neuronal exosomes facilitate synaptic pruning by up-regulating complement factors in microglia. Scientific Reports, $2015,5,7989$.

79. Bátiz, L. F.; Castro, M. A.; Burgos, P. V.; Velásquez, Z. D.; Muñoz, R. I.; Lafourcade, C. A.; Troncoso-Escudero, P.; Wyneken, U. Exosomes as Novel Regulators of Adult Neurogenic Niches. Frontiers in cellular neuroscience, 2016, 9, 501.

80. Malm, T.; Loppi, S.; Kanninen, K. M. Exosomes in Alzheimer's disease. Neurochemistry International, 2016, 97, 193-199.

81. Grapp, M.; Wrede, A.; Schweizer, M.; Huwel, S.; Galla, HJ.; Snaidero, N.; Simons, M.; Buckers, J.; Low, P.S.; Urlaub, H.; Gartner, J.; Steinfeld, R.; Choroid plexus transcytosis and exosome shuttling deliver folate into brain parenchyma. Nature Communications, 4 (2013), 2123.

82. Zabeo, D.; Cvjetkovic, A.; Lässer, C.; Schorb, M.; Lötvall, J.; Höög, J. L. Exosomes purified from a single cell type have diverse morphology. Journal of extracellular vesicles, 2017 6(1), 1329476.

83. Yu, L.L.; Zhu, J.; Liu J-X.; Jiang, F.; Ni, W.K.; Qu, L.S.; Ni, R.Z.; Lu, C.H.; Xiao M.B.. A Comparison of Traditional and Novel Methods for the Separation of Exosomes from Human Samples. Biomed Research International, 2018, 1-9.

84. Li, P.; Kaslan, M.; Lee, S. H.; Yao, J.; Gao, Z. Progress in exosome isolation techniques. Theranostics, 2017, 7, 789-804.

85. Sharples, R. A.; Scicluna, B. J.; Hill, A. F. Exosomes provide a protective and enriched source of miRNA for biomarker profiling compared to intracellular and cellfree blood AU - Cheng, Lesley. J. Extracell. Vesicles 2014, 3, 23743.

86. Lötvall, J.; Hill, A. F.; Hochberg, F.; Buzás, E. I.; Di Vizio, D.; Gardiner, C.; Gho, Y. S.; Kurochkin, I. V.; Mathivanan, S.; Quesenberry, P.; Sahoo, S.; Tahara, H.; Wauben, M. H.; Witwer, K. W.; \& Théry, C. Minimal experimental requirements for definition of extracellular vesicles and their functions: a position statement from the International Society for Extracellular Vesicles. Journal of extracellular vesicles, 2014, 3, 26913.

87. Fauré, J.; Lachenal, G.; Court, M.; Hirrlinger, J.; Chatellard-Causse, C.; Blot, B.; Grange, J.; Schoehn, G.; Goldberg, Y.; Boyer, V.; Kirchhoff, F.; Raposo, G.; Garin, J.; Sadoul, R. Exosomes are released by cultured cortical neurones. Molecular and Cellular Neurosciences, 2006, 31(4):642-8.

88. Lachenal, G.; Pernet-Gallay, K.; Chivet, M.; Hemming, F.J.; Belly, A.; Bodon, G.; Blot, B.; Haase, G.; Goldberg, Y.; Sadoul R. Release of exosomes from differentiated neurons and its regulation by synaptic glutamatergic activity. Molecular and Cellular Neurosciences, 2011, 46(2), 409-18.

89. Krämer-Albers, E.M.; Bretz, N.; Tenzer, S.; Winterstein, C.; Möbius, W.; Berger, H.; Nave K.A.; Schild, H.; Trotter J. Oligodendrocytes secrete exosomes containing major myelin and stress-protective proteins: Trophic support for axons? Proteomics Clinical Applications, 2007, (11), 1446-61. 


\section{Captions for Figures}

4 Figure 1: Microglia activation with release of extracellular vesicles (EVs). 5 lipopolysaccharide (LPS), mitogen-activated protein kinases (MAPKs) superfamily, c-Jun N-

6 terminal kinase (JNK 1/2) and p38 proteins, nuclear factor- $\kappa \mathrm{B}(\mathrm{NF}-\kappa \mathrm{B})$ interleukin (IL)-1 $\beta$, 7 IL-6 and tumour necrosis factor (TNF)- $\alpha$.

\section{Captions for Graphical Abstract}

9 Biosynthesis and release of extracellular vesicles: 